Article

\title{
Pharmacological Inhibition of CCR2 Signaling Exacerbates Exercise-Induced Inflammation Independently of Neutrophil Infiltration and Oxidative Stress
}

\author{
Takaki Tominaga ${ }^{1,2, *(\mathbb{C})}$, Jiapeng Huang ${ }^{1}$ and Katsuhiko Suzuki ${ }^{3}$ (i) \\ 1 Graduate School of Sport Sciences, Waseda University, Tokorozawa 359-1192, Japan; \\ hjpshidsg1234@toki.waseda.jp \\ 2 Japan Society for the Promotion of Sciences, Tokyo 102-0083, Japan \\ 3 Faculty of Sport Sciences, Waseda University, Tokorozawa 359-1192, Japan; katsu.suzu@waseda.jp \\ * Correspondence: takaki.k-bbc@akane.waseda.jp; Tel.: +81-4-2947-6753
}

Citation: Tominaga, T.; Huang, J.;

Suzuki, K. Pharmacological

Inhibition of CCR2 Signaling

Exacerbates Exercise-Induced

Inflammation Independently of

Neutrophil Infiltration and Oxidative

Stress. Immuno 2022, 2, 26-39.

https://doi.org/10.3390/

immuno2010003

Received: 2 November 2021

Accepted: 22 December 2021

Published: 27 December 2021

Publisher's Note: MDPI stays neutral with regard to jurisdictional claims in published maps and institutional affiliations.

Copyright: (c) 2021 by the authors Licensee MDPI, Basel, Switzerland. This article is an open access article distributed under the terms and conditions of the Creative Commons Attribution (CC BY) license (https:// creativecommons.org/licenses/by/ $4.0 /)$.

\begin{abstract}
Although exercise-induced humoral factors known as exerkines benefit systemic health, the role of most exerkines has not been investigated. Monocyte chemoattractant protein-1 (MCP-1) is a representative chemokine whose circulating concentrations increase after exercise, and it is one of the exerkines. MCP-1 is a ligand for CC chemokine receptor 2 (CCR2), which is expressed on monocytes, macrophages, and muscle cells. However, there is no information on the role of CCR2 signaling in exercise. Therefore, to investigate the research question, we administrated CCR2 antagonist or PBS to mice to inhibit CCR2 signaling before and after exercise. Our results showed that CCR2 signaling inhibition promoted exercise-induced macrophage infiltration and inflammation $24 \mathrm{~h}$ after exercise in muscle. CCR2 signaling inhibition also exacerbated exercise-induced inflammation immediately after exercise in muscle. However, neutrophil infiltration and oxidative stress had no contribution to exercise-induced inflammation by CCR2 signaling inhibition. CCR2 signaling inhibition also exacerbated exercise-induced inflammation immediately after exercise in kidney, liver, and adipose tissues. To summarize, pharmacological inhibition of CCR2 signaling exacerbated exercise-induced inflammation independently of neutrophil infiltration and oxidative stress.
\end{abstract}

Keywords: CC chemokine receptor 2 (CCR2); exercise; macrophage; inflammation; monocyte chemoattractant protein-1 (MCP-1)

\section{Introduction}

Regular physical activity has preventative effects against various diseases (e.g., type 2 diabetes, stroke, cancer, and dementia) [1,2]. A single bout of exercise also benefits systemic health (e.g., stimulation of glucose uptake independently of insulin signaling, improvement of insulin sensitivity, and cognitive function) [3,4]. Recent research has shown that exercise-induced humoral factors known as exerkines contribute to these exercise effects [2]. However, the role of most exerkines has not been investigated. Therefore, it is important to elucidate the role of each exerkine.

Strenuous exercise induces systemic inflammation and increases the circulating concentrations of various inflammatory mediators [5-9]. Among the inflammatory mediators such as cytokines and chemokines whose circulating concentrations fluctuate by exercise, monocyte chemoattractant protein-1 (MCP-1) is a representative chemokine whose concentrations increase following exercise in circulation, urine, and muscles [5-12]. MCP-1 is also secreted from $\mathrm{C} 2 \mathrm{C} 12$ myotubes upon electric pulse stimulation, which induces muscle contraction and mimics exercise [13-15]. These studies suggest that MCP-1 is one of the exerkines.

MCP-1 exerts chemotactic activity for monocytes/macrophages that remove and repair damaged muscle fibers $[16,17]$. Following exercise, it has been suggested that MCP-1 is an 
important mediator for macrophage infiltration in muscle, which contributes to muscle damage and inflammation [10-12]. MCP-1 is a ligand for CC chemokine receptor 2 (CCR2), which is expressed on monocytes/macrophages [16,17]. Several studies have reported that in various muscle injury models, CCR2 is essential for macrophage accumulation in muscle [16-18]. Therefore, CCR2 signaling may also be an important factor for exerciseinduced macrophage infiltration and inflammation. CCR2 is also expressed on muscle cells, myogenic progenitor cells, and satellite cells, and CCR2 signaling contributes to muscle insulin resistance and dysfunction of myogenic progenitor cells [18-21]. Therefore, muscle cells may also be other targets for CCR2 ligands. However, the role of CCR2 signaling in endurance exercise has not been investigated.

Understanding the molecular mechanisms of exercise-induced inflammation can be helpful in developing appropriate countermeasures (e.g., functional food intake, meal intervention, and pharmacological approaches). In the present study, we hypothesized that CCR2 signaling is essential for macrophage accumulation and regulation of inflammation following exercise. To confirm this hypothesis, we administrated CCR2 antagonist to inhibit CCR2 signaling before and after exercise. In contrast to our expectations, we observed that pharmacological inhibition of CCR2 signaling exacerbates exercise-induced macrophage infiltration and inflammation in muscle, kidney, liver, and adipose tissues.

\section{Materials and Methods}

\subsection{Animals}

Male C57BL/6J mice (aged 10-11 weeks) purchased from Takasugi Experimental Animals Supply (Kasukabe, Japan) were used in this study. They were housed in the breeding room under a 12-h light/dark cycle. The mice had free access to standard chow (MF, oriental yeast, Tokyo, Japan) and water. The experimental procedures were approved (2020-A29) and conducted according to the Guiding Principles for the Care and Use of Animals in the Academic Research Ethical Review Committee of Waseda University.

\subsection{Experimental Protocols}

To investigate the role of CCR2 signaling $24 \mathrm{~h}$ after exercise, the mice were randomly divided into four groups as follows: rest with PBS administration, rest with CCR2 antagonist administration, exercise with PBS administration and exercise with CCR2 antagonist administration. The CCR2 antagonist RS-504393 (Abcam, Cambridge, U.K.) was dissolved in PBS (100 $\mu$ g in $100 \mu \mathrm{L}$ of PBS; approximately $4 \mathrm{mg} / \mathrm{kg}$ body weight), or $100 \mu \mathrm{L}$ of PBS was administered orally $1 \mathrm{~h}$ before and $12 \mathrm{~h}$ after the first administration. The exercised mice were sacrificed $24 \mathrm{~h}$ after the exercise, and the rest mice were sacrificed in the same time course as the exercised mice ( $n=6$, per group).

To investigate the role of CCR2 signaling immediately after exercise, mice were randomly divided into four groups in the same manner. Dissolved RS-504393 $(100 \mu \mathrm{g}$ in $100 \mu \mathrm{L}$ of PBS; approximately $4 \mathrm{mg} / \mathrm{kg}$ body weight) or $100 \mu \mathrm{L}$ of PBS was administered orally $1 \mathrm{~h}$ before exercise. The exercised mice were sacrificed immediately after the exercise, and the rest mice were sacrificed in the same time course as the exercise mice ( $n=6$, per group).

Four days before the experiments, all mice were accustomed to treadmill running at $15 \mathrm{~m} / \mathrm{min}$ and $7 \%$ grade for $10 \mathrm{~min}$. On the experimental days, the mice were subjected to treadmill running at $24 \mathrm{~m} / \mathrm{min}$ and $7 \%$ grade for $60 \mathrm{~min}$. Heparinized blood samples were collected from the abdominal aorta under isoflurane inhalation anesthesia (Abbott, Tokyo, Japan), and then the mice were perfused by PBS to remove blood in the tissues. After cervical dislocation, the gastrocnemius, kidney, liver, and epididymal adipose tissues were harvested and frozen in liquid nitrogen. Plasma samples were obtained from blood centrifuged at $1600 \times \mathrm{g}$ for $10 \mathrm{~min}$ at $4{ }^{\circ} \mathrm{C}$. All samples were stored at $-80^{\circ} \mathrm{C}$ until analysis.

\subsection{Real-Time PCR}

Total RNA was extracted from the gastrocnemius, kidney, liver, and adipose tissues using TRIZOL Reagent (Invitrogen, Carlsbad, CA, USA). The purity and concentrations of 
the extracted total RNA were measured using NanoDrop 1000 (Thermo Fisher Scientific, Waltham, MA, USA). The total RNA was reverse transcribed to cDNA using the HighCapacity cDNA Reverse Transcription Kit (Applied Biosystems, Foster City, CA, USA). PCR was performed using the Fast 7500 Real-time PCR system (Applied Biosystems, Foster City, CA, USA) and Fast SYBR Green Master Mix (Applied Biosystems, Foster City, CA, USA). The PCR conditions for all genes consisted of one denaturation cycle at $95^{\circ} \mathrm{C}$ for $30 \mathrm{~s}$, 40 cycles consisting of denaturing at $95^{\circ} \mathrm{C}$ for $3 \mathrm{~s}$, and annealing and elongation at $60^{\circ} \mathrm{C}$ for $30 \mathrm{~s}$. Each gene was normalized using $18 \mathrm{~s}$ rRNA. Because the difference in kidney $\beta$-actin expression between groups was smaller than $18 \mathrm{~s}$ rRNA expression (data not shown), we used $\beta$-actin for the normalization of kidney gene expression. Although $18 \mathrm{~s}$ rRNA expression was significantly different between groups in some tissues, the maximal fold change of each housekeeping gene expression between groups was 1.3 times (Supplemental Figure S1). Therefore, we concluded that the effects of normalization using $18 \mathrm{~s}$ rRNA or $\beta$-actin are small. All data were calculated using the $\Delta \Delta \mathrm{Ct}$ method. The specific primer sequences are shown in Table 1.

Table 1. Primer sequences for Real time-PCR.

\begin{tabular}{|c|c|c|}
\hline & Forward & Reverse \\
\hline $18 \mathrm{~s}$ & TTCTGGCCAACGGTCTAGACAAC & CCAGTGGTCTTGGTGTGCTGA \\
\hline Arginase1 & CTCCAAGCCAAAGTCCTTAGAG & AGGAGCTGTCATTAGGGACATC \\
\hline ATF4 & AACCTCATGGGTTCTCCAGCGA & CTCCAACATCCAATCTGTCCCG \\
\hline$\beta$-actin & GCGGACTGTTACTGAGCTGCGT & TGCTGTCGCCTTCACCGTTCC \\
\hline CAT & ACATGGTCTGGGACTTCTGG & CAAGTTTTTGATGCCCTGGT \\
\hline CCR2 & ACAGCTCAGGATTAACAGGGACTTG & ACCACTTGCATCCACACATGAC \\
\hline CCR5 & CATCCGTTCCCCCTACAAGA & GGAACTGACCCTTGAAAATCCA \\
\hline CD11c & CTGGATAGCCTTTCTTCTGCTG & GCACACTGTGTCCGAACTC \\
\hline CD163 & GGGTCATTCAGAGGCACACTG & CTGGCTGTCCTGTCAAGGCT \\
\hline CD206 & CAAGGAAGGTTGGCATTTGT & CСTTTCAGTCCTTTGCAAGC \\
\hline CD68 & CTTCCCACAGGCAGCACAG & AATGATGAGAGGCAGCAAGAGG \\
\hline $\mathrm{CHOP}$ & TATCTCATCCCCAGGAAACG & TATCTCATCCCCAGGAAACG \\
\hline CX3CL1 & ACGAAATGCGAAATCATGTGC & CTGTGTCGTCTCCAGGACAA \\
\hline $\mathrm{F} 4 / 80$ & CTTTGGCTATGGGCTTCCAGTC & GCAAGGAGGACAGAGTTTATCGTG \\
\hline GSTm3 & GCTCTTACCACGTGCAGCTT & GGCTGGGAAGAGGAAATGGA \\
\hline HO-1 & CACGCATATACCCGCTACCT & CCAGAGTGTTCATTCGAGCA \\
\hline IL-10 & CGCAGCTCTAGGAGCATGTG & GCTCTTACTGACTGGCATGAG \\
\hline IL-1 $\beta$ & GGGCCTCAAAGGAAAGAATC & TTGCTTGGGATCCACACTCT \\
\hline IL-4 & GGTCTCAACCCCCAGCTAGT & GCCGATGATCTCTCTCAAGTGAT \\
\hline IL-6 & AACGATGATGCACTTGCAGA & TGGTACTCCAGAAGACCAGAGG \\
\hline MCP-1 & CTTCTGGGCCTGCTGTTCA & CCAGCCTACTCATTGGGATCA \\
\hline MCP-2 & AGAGACAGCCAAAGCTGGAA & CAGGCACCATCTGCTTGTAA \\
\hline MCP-3 & CACATTCCTACAGACAGCTC & AGCTACAGAAGGATCACCAG \\
\hline MIP-1 $\alpha$ & ACTGCCTGCTGCTTCTCCTACA & ATGACACCTGGCTGGGAGCAAA \\
\hline MIP-1 $\beta$ & ACССТСССАСТTССТGСTGTTT & CTGTCTGCCTCTTTTGGTCAGG \\
\hline NADPH Oxidase & TTGGGTCAGCACTGGCTCTG & TGGCGGTGTGCAGTGCTATC \\
\hline
\end{tabular}


Table 1. Cont.

\begin{tabular}{crc}
\hline & Forward & Reverse \\
\hline NQO1 & GGTATTACGATCCTCCCTCAACATC & GAGTACCTCCCATCCTCTCTTCTTC \\
\hline Nrf1 & GTGGGACAGCAAGCGATTGTAC & CGCACCACATTCTCCAAAGG \\
\hline Nrf2 & CTCGCTGGAAAAAGAAGTGG & CCGTCCAGGAGTTCAGAGAG \\
\hline ORP150 & CAGACTGAAGAGGCGAAACC & TTCCTGTTCAGGTCCAGCTC \\
\hline PGC-1 $\alpha$ & AGCCGTGACCACTGACAACGAG & GCTGCATGGTTCTGAGTGCTAAG \\
\hline Sirt1 & GCAACAGCATCTTGCCTGAT & GTGCTACTGGTCTCACTT \\
\hline SOD1 & GAGACCTGGGCAATGTGACT & GTTTACTGCGCAATCCCAAT \\
\hline SOD2 & TCAAGCGTGACTTTGGGTCT & AGCGGAATAAGGCCTGTTGT \\
\hline TNF- $\alpha$ & CCTCCCTCTCATCAGTTCTA & ACTTGGTGGTTTGCTACGAC
\end{tabular}

\subsection{Histological Analysis}

Cross sections of $10 \mu \mathrm{m}$ frozen gastrocnemius were used for hematoxylin and eosin (H\&E) staining and immunohistochemistry. For immunohistochemistry, the sections were first fixed in $4 \%$ paraformaldehyde for $7 \mathrm{~min}$ and then incubated with $5 \%$ bovine serum albumin (BSA) in PBS for $30 \mathrm{~min}$. Next, the sections were incubated overnight at $4{ }^{\circ} \mathrm{C}$ with primary antibodies in PBS with 5\% BSA. After washing the sections, they were incubated with the secondary antibodies in PBS with 5\% BSA for $1 \mathrm{~h}$ at room temperature. After washing, the sections were mounted using the Vectashield mounting medium (H-1800, Vector Laboratories, Burlington, ON, Canada) containing DAPI to visualize the nuclei. The antibodies used in this study are shown in Table 2. The stained sections were observed under a fluorescence microscope BZ-8000 (KEYENCE, Osaka, Japan). Three fields at $\times 200$ magnification were captured per animal to identify the localization of IL-6. The number of M1 and M2 macrophages was quantified according to a previous study [22]. Briefly, we defined M1 macrophages as F4/80 ${ }^{+} \mathrm{CD}_{206}{ }^{-}$cells and M2 macrophages as F4/80 ${ }^{+} \mathrm{CD} 206^{+}$ cells in the same section. Two to four fields at $\times 100$ magnification were captured per animal to quantify the number of macrophages. We then calculated the average value of each field as the measured data.

Table 2. Antibodies for immunohistochemistry.

\begin{tabular}{|c|c|c|c|}
\hline Antibodies & Source & Identifier & Dilution (Concentration) \\
\hline Goat anti-IL-6 antibody & R\&D Systems, Minneapolis, MN, USA & Cat\# AF406 & $10 \mu \mathrm{g} / \mathrm{mL}$ \\
\hline $\begin{array}{l}\text { Mouse anti-dystrophin antibody } \\
\text { (clone 1808) }\end{array}$ & Abcam, Cambridge, UK & Cat\# ab3149 & $1: 400$ \\
\hline $\begin{array}{l}\text { Rat anti-F4/80 antibody } \\
\text { (clone BM8) }\end{array}$ & Biolegend, San Diego, CA, USA & Cat\# 123101 & $1: 100$ \\
\hline Goat anti-CD206 antibody & R\&D Systems, Minneapolis, MN, USA & Cat\# AF2535 & $2 \mu \mathrm{g} / \mathrm{mL}$ \\
\hline $\begin{array}{c}\text { Alexa Fluor 555-conjugated rabbit } \\
\text { anti-goat IgG antibody }\end{array}$ & Thermo Fisher Scientific, Waltham, MA, USA & Cat\# A21431 & $1: 200$ \\
\hline $\begin{array}{l}\text { Fluorescein-conjugate horse } \\
\text { anti-mouse IgG antibody }\end{array}$ & Vector Laboratories, Burlington, ON, Canada & Cat\# FI-2000 & $1: 200$ \\
\hline $\begin{array}{l}\text { Alexa Fluor 488-conjugated donkey } \\
\text { anti-rat IgG antibody }\end{array}$ & Thermo Fisher Scientific, Waltham, MA, USA & Cat\# A21208 & $1: 200$ \\
\hline $\begin{array}{l}\text { Alexa Fluor 555-conjugated donkey } \\
\text { anti-goat IgG antibody }\end{array}$ & Abcam, Cambridge, UK & $\begin{array}{c}\text { Cat\# } \\
\text { ab150130 }\end{array}$ & $1: 200$ \\
\hline
\end{tabular}




\subsection{ELISA and TBARS Assay}

Plasma and tissue MCP-1 concentrations were measured using the Mouse MCP-1 DuoSet ELISA kit (R\&D Systems, Minneapolis, MN, USA), tissue IL- $1 \beta$ concentrations were measured using the Mouse IL-1 $\beta$ DuoSet ELISA kit (R\&D Systems, Minneapolis, $\mathrm{MN}, \mathrm{USA}$ ), and tissue myeloperoxidase (MPO) concentrations were measured using the MPO ELISA kit (Hycult Biotech, Uden, The Netherlands). Gastrocnemius thiobarbituric acid reactive substance (TBARS) concentrations were measured using the TBARS assay kit (Cayman Chemical Co., Ann Arbor, MI, USA). To measure the tissue concentrations of MCP-1, IL-1 $\beta$, MPO, and TBARS, the tissue was homogenized in the tissue protein extraction reagent (T-PER; Thermo Fisher Scientific, Waltham, MA, USA) containing a protease inhibitor (complete mini protease inhibitor cocktail tablet; Roche Diagnostics, Mannheim, Germany). The homogenate was centrifuged at $10,000 \times \mathrm{g}$ for $15 \mathrm{~min}$ at $4{ }^{\circ} \mathrm{C}$ and the supernatant was collected. To avoid tissue debris contamination, we repeated the centrifugation step. Total protein concentrations in the supernatant were measured using the Pierce ${ }^{\mathrm{TM}}$ BCA Protein Assay Kit (Thermo Fisher Scientific, Waltham, MA, USA). The supernatant was used for each assay. The tissue concentrations of MCP-1, IL-1 $\beta$, MPO, and TBARS were normalized using the total protein in the supernatant. The absorbance was measured using Spectra Max iD5 (Molecular Devices Inc., San Jose, CA, USA).

\subsection{Statistical Analysis}

Data are expressed as mean \pm standard error (SE). Two-way analysis of variance (ANOVA) followed by Bonferroni post hoc test was used for analyzing data. Statistical analysis was performed using SPSS V25.0 (IBM Japan, Ltd., Tokyo, Japan), with statistical significance being defined as $p<0.05$.

\section{Results}

3.1. Inhibition of CCR2 Signaling Exacerbates Macrophage Infiltration and Inflammation $24 \mathrm{~h}$ after Exercise in Muscle

We had previously reported that exercise induces macrophage infiltration in muscle, which contributes to exercise-induced inflammation [10-12,22]. In several studies, we observed macrophage infiltration $24 \mathrm{~h}$ after exercise [11,12,22]. To investigate the role of CCR2 signaling in exercise-induced macrophage infiltration and inflammation in muscle, we administrated CCR2 antagonist to inhibit CCR2 signaling before and after exercise, and the gastrocnemius was excised $24 \mathrm{~h}$ after exercise. In contrast to our expectation, exercise with CCR2 antagonist administration induced the gene expression of a pan-macrophage marker (F4/80), M1 macrophage markers (CD68, CD11c), an M2 macrophage marker (arginase 1), and chemokine receptors expressed on M1 macrophages (CCR2, CCR5) $[17,18,23]$ (Figure 1A). The histological analysis revealed that exercise with CCR2 antagonist administration promoted the infiltration of M1 macrophages (F4/80 $\mathrm{CD}^{2} 6^{-}$cells) (Figure 1B,C). However, exercise with PBS administration did not induce such changes (Figure 1A-C). The histological analysis also revealed that the infiltration of M2 macrophages (F4/80 $0^{+} \mathrm{CD} 206^{+}$cells) did not change after exercise with and without CCR2 antagonist administration (Figure 1B,D). Muscle injury induces immune cell infiltration and inflammation [16-18,22]. However, we did not observe abnormal muscle fibers (e.g., necrotic fibers) in all groups (Figure 1E). M1 macrophages produce inflammatory cytokines and chemokines [17]. We next investigated the local inflammation. Exercise with CCR2 antagonist administration induced the gene expression of cytokines and chemokines such as interleukin- $1 \beta$ (IL-1 $\beta$ ), tumor necrosis factor- $\alpha$ (TNF- $\alpha$ ), IL-10, MCP-1, MCP-3, and macrophage inflammatory protein-1 $\beta$ (MIP-1 $\beta$ ) (Figure 1F). However, exercise with PBS administration did not induce such changes (Figure 1F). We next measured the concentrations of plasma MCP-1 to investigate whether inhibition of CCR2 signaling exacerbates systemic inflammation. However, plasma MCP-1 concentrations showed no changes after exercise with and without CCR2 antagonist administration (Figure 1G). These results indicated that 
the inhibition of CCR2 signaling exacerbated exercise-induced M1 macrophage infiltration and inflammation at a local level $24 \mathrm{~h}$ after exercise.

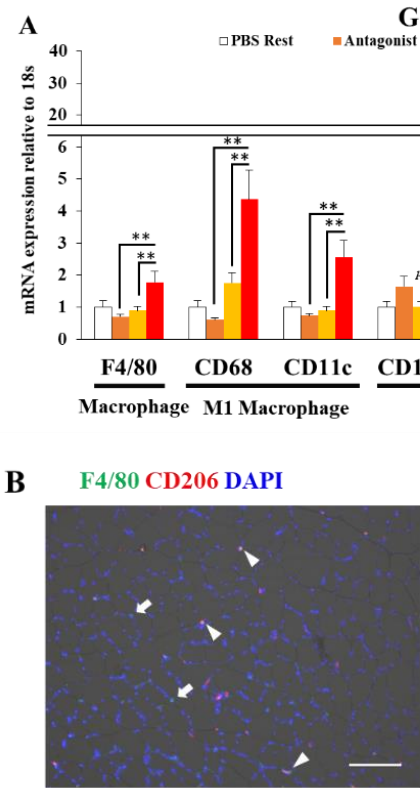

PBS Rest

$\mathbf{E}$

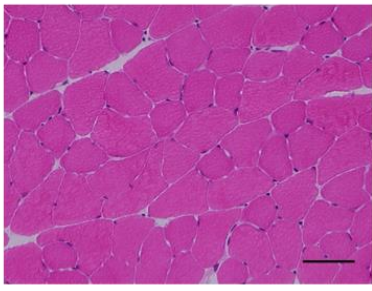

PBS Rest

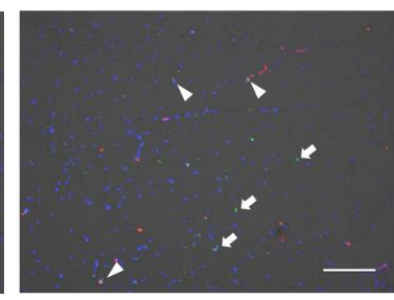

Antagonist Rest

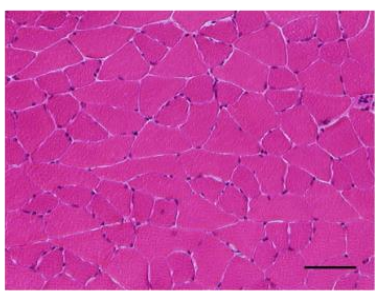

Antagonist Rest

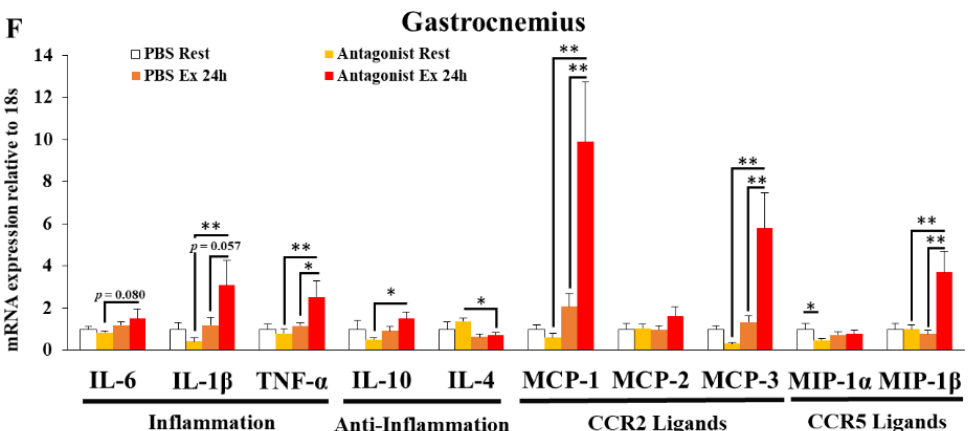

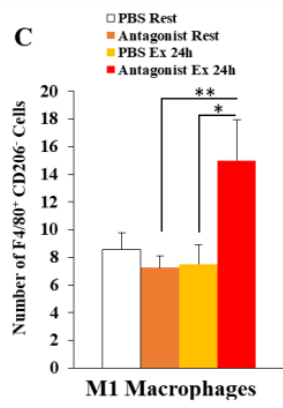

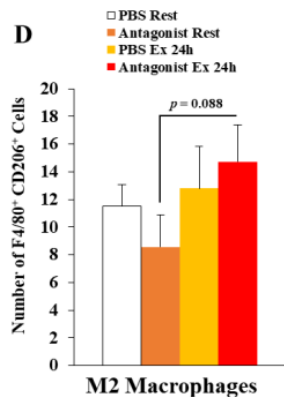

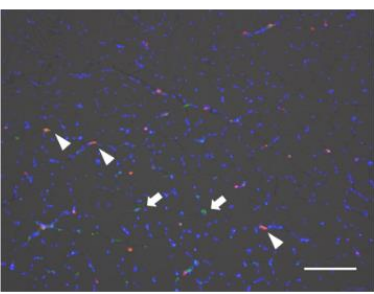

PBS Ex 0h

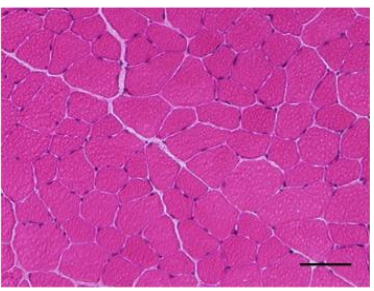

PBS Ex 24h

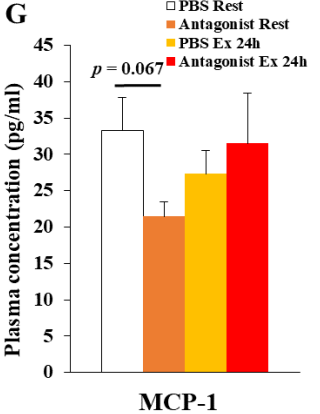

Figure 1. (A) Gene expression of macrophage markers $(n=5-6)$. (B) Representative images of gastrocnemius sections using immunofluorescence staining (green, F4/80; red, CD206; blue, DAPI; magnification $\times 100, n=5-6)$. Images were merged with bright field. Arrows indicate M1 macrophages (F4/80 ${ }^{+}$ CD206 ${ }^{-}$cells, $\left.n=5-6\right)$. Arrowheads indicate M2 macrophages (F4/80 ${ }^{+}$CD206 $6^{+}$cells, $\left.n=5-6\right)$. Scale bar is $100 \mu \mathrm{m}$. (C) The number of M1 macrophages per field. (D) The number of M2 macrophages per field. (E) Representative images of gastrocnemius sections using H\&E staining (magnification $\times 200, n=3$ ). Arrowheads indicate immune cell infiltration. Scale bar is $50 \mu \mathrm{m}$. (F) Gene expression of inflammation $(n=5-6)$. (G) Plasma MCP-1 concentrations $(n=5-6)$. Data are shown as mean \pm SE. Data were analyzed using two-way ANOVA followed by Bonferroni post hoc test ${ }^{*} p<0.05$, ** $p<0.01)$. Arg1, Arginase1; CCR, CC chemokine receptor; IL, interleukin; TNF, tumor necrosis factor; MCP, monocyte chemoattractant protein; MIP, macrophage inflammatory protein; ANOVA, analysis of variance; $\mathrm{SE}$, standard error. 
3.2. Inhibition of CCR2 Signaling Exacerbates Exercise-Induced Inflammation Immediately after Exercise Independently of Neutrophil Infiltration in Muscle

We next investigated the role of CCR2 signaling in inflammation immediately after exercise in muscle. Exercise with CCR2 antagonist administration induced the gene expression of cytokines and chemokines such as IL- 6 , IL-1 $\beta$, TNF- $\alpha$, IL-10, MCP-3, and MIP-1 $\beta$, but not the macrophage marker, F4/80 (Figure 2A). Exercise with CCR2 antagonist administration also induced the increase in IL-1 $\beta$ protein concentrations (Figure $2 \mathrm{~B}$ ). However, exercise with PBS administration did not induce such changes (Figure 2A,B). Moreover, plasma MCP-1 concentrations, as an indicator of systemic inflammation, showed no changes after exercise (Figure 2C). These results indicated that the inhibition of CCR2 signaling exacerbated exercise-induced inflammation at a local level immediately after exercise. We also did not observe necrotic muscle fibers in all groups (Figure 2D), which suggested that necrosis of muscle fibers does not cause this inflammation.

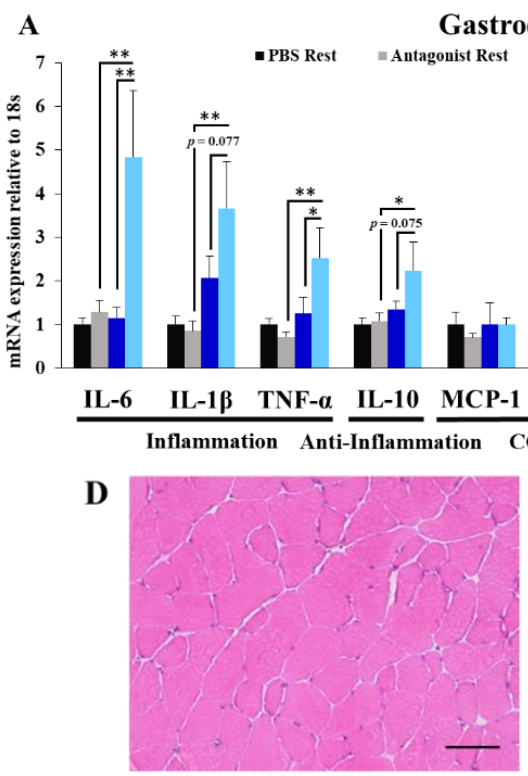

PBS Rest

F Dystrophin IL-6 DAPI

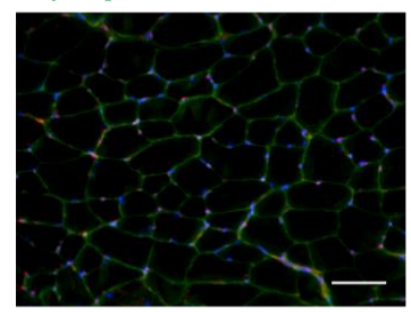

PBS Rest
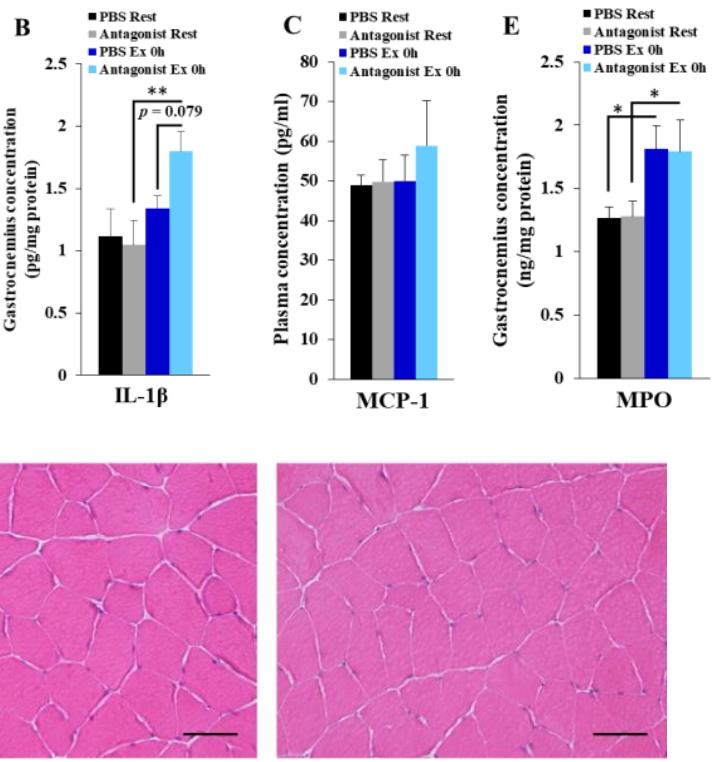

Antagonist Ex 0h

PBS Ex 0h

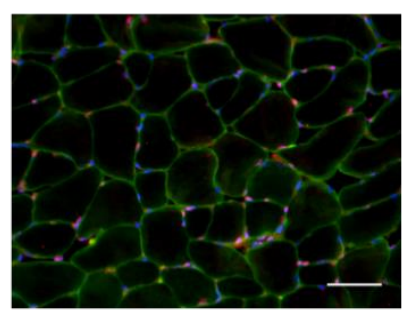

Antagonist Rest

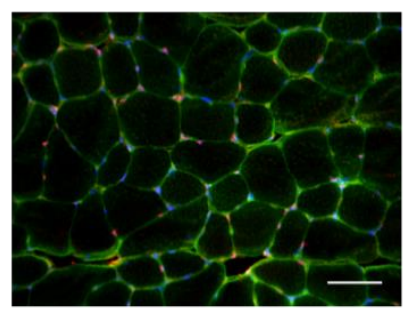

PBS Ex 0h

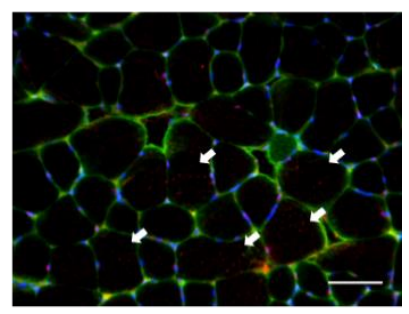

Antagonist Ex 0h

Figure 2. (A) Gene expression of inflammation $(n=5-6)$. (B) Gastrocnemius IL- $1 \beta$ concentrations $(n=6)$. (C) Representative images of gastrocnemius sections using immunofluorescence staining (green, dystrophin; red, IL-6; blue, DAPI; magnification $\times 200, n=2$ ). Arrowheads indicate IL-6 positive muscle fibers. Scale bar is $50 \mu \mathrm{m}$. (D) Representative images of gastrocnemius sections using H\&E staining (magnification $\times 200, n=2$ ). Scale bar is $50 \mu \mathrm{m}$. (E) Plasma MCP-1 concentrations $(n=5-6)$. (F) Gastrocnemius MPO concentrations $(n=6)$. Data are shown as mean $\pm \mathrm{SE}$. Data were analyzed using two-way ANOVA followed by Bonferroni post hoc test $\left(^{*} p<0.05,{ }^{* *} p<0.01\right)$. IL, interleukin; TNF- $\alpha$, tumor necrosis factor- $\alpha$; MCP, monocyte chemoattractant protein; MIP, macrophage inflammatory protein; MPO, myeloperoxidase; ANOVA, analysis of variance; SE, standard error.

We have previously reported that neutrophils contribute to exercise-induced inflammation in muscle [11]. Therefore, we hypothesized that neutrophil infiltration contributes to exercise-induced inflammation by the inhibition of CCR2 signaling. We found that 


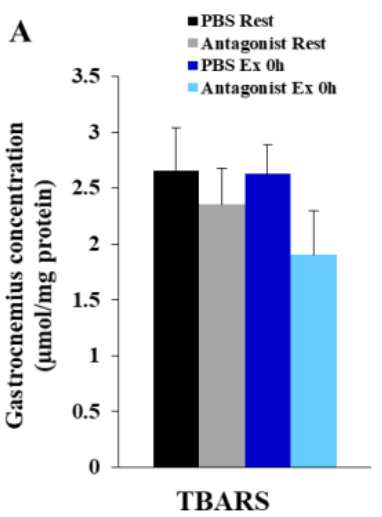

exercise with and without CCR2 antagonist administration increased the concentrations of gastrocnemius MPO, a neutrophil infiltration marker (Figure 2E). However, the gastrocnemius MPO concentrations showed no difference between exercise with and without CCR2 antagonist administration (Figure 2E). This result suggested that CCR2 signaling inhibition exacerbated exercise-induced inflammation independently of neutrophil infiltration.

We next investigated the localization of IL-6 to identify the source of inflammatory cytokine. The histological analysis revealed that IL-6 is primarily localized in the interfiber space (Figure 2F). However, exercise with CCR2 antagonist administration also induced IL-6 expression in muscle fibers, but the signal was weak compared to that in the interfiber space (Figure 2F). This result suggested that muscle fibers may contribute to exercise-induced inflammation by inhibition of CCR2 signaling.

\subsection{Inhibition of CCR2 Signaling Does Not Influence Muscle Oxidative Stress Immediately after} Exercise

Oxidative stress is one of the triggers of exercise-induced inflammation [7]. Hence, we next hypothesized that oxidative stress contributes to exercise-induced inflammation by the inhibition of CCR2 signaling. However, we observed that there were no changes in the concentrations of gastrocnemius TBARS, an oxidative stress marker, by exercise with and without CCR2 antagonist administration (Figure 3A). Moreover, the gene expression of the oxidative enzyme NADPH oxidase showed no changes (Figure 3B). These results indicated that oxidative stress does not contribute to exercise-induced inflammation by the inhibition of CCR2 signaling. Because NF-E2-related factor 2 (Nrf2) is known to activate anti-inflammatory systems [24,25], we hypothesized that Nrf2 is an important factor of exercise-induced inflammation by inhibiting CCR2 signaling. Nrf2 gene expression was increased by exercise with and without CCR2 antagonist administration (Figure 3B). However, the downstream genes of Nrf2 such as heme oxygenase-1 (HO-1), glutathione S-transferase (GST), and NADPH quinone dehydrogenase-1 (NQO-1) [24,25] exhibited inconsistent changes (Figure 3B). These results indicated that the Nrf2 pathway did not activate and may not contribute to exercise-induced inflammation by the inhibition of CCR2 signaling.

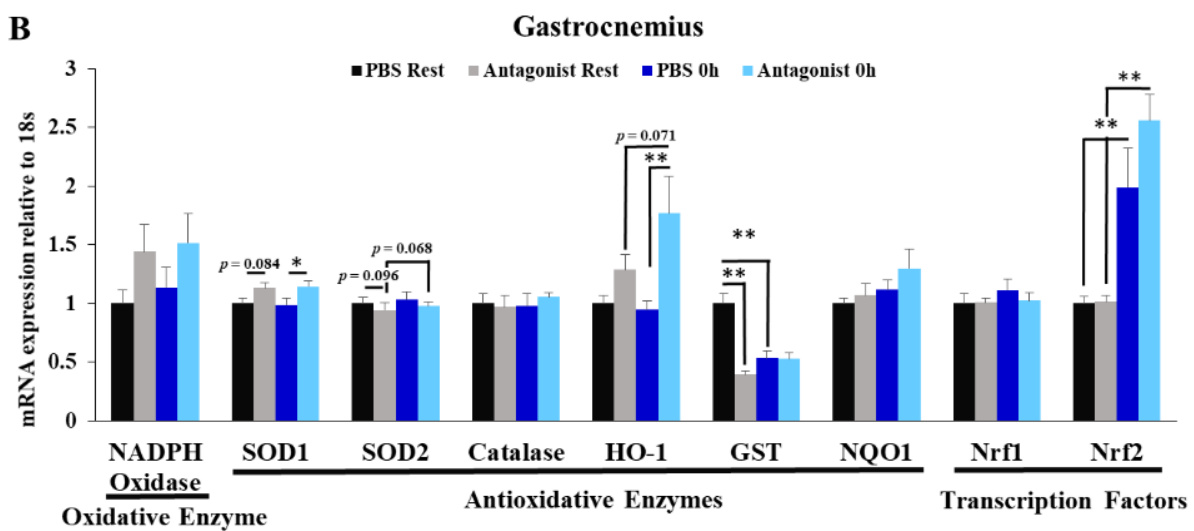

Figure 3. (A) Gastrocnemius TBARS concentrations $(n=6)$. (B) Gene expression of an oxidative enzyme, antioxidative enzymes and transcription factors $(n=6)$. Data are shown as mean $\pm \mathrm{SE}$. Data were analyzed using two-way ANOVA followed by Bonferroni post hoc test $\left({ }^{*} p<0.05, * * p<0.01\right)$. TBARS, thiobarbituric acid reactive substance; SOD, superoxide dismutase; HO-1, heme oxygenase-1; GST, glutathione S-transferase; NQO, NADPH quinone dehydrogenase; Nrf, NF-E2-related factor; ANOVA, analysis of variance; SE, standard error. 
3.4. Effects of CCR2 Signaling Inhibition on Muscle Gene Expression of ER Stress Marker and Exercise Adaptation-Related Genes Immediately after Exercise

Endoplasmic reticulum (ER) stress is known to induce inflammation [26]; therefore, we next hypothesized that ER stress is an important factor of exercise-induced inflammation by inhibiting CCR2 signaling. The gene expression of the ER stress marker oxygenregulated protein 150 (ORP150) was induced by exercise with CCR2 antagonist administration (Figure 4). However, the expression of other markers activating transcription factor 4 (ATF4) and C/EBP homologous protein (CHOP) did not increase by exercise (Figure 4). These results indicated that ER stress may not strongly contribute to exercise-induced inflammation by the inhibition of CCR2 signaling. The expression of exercise adaptationrelated genes such as peroxisome proliferator-activated receptor coactivator-1 $\alpha$ (PGC-1 $\alpha)$ and sirtuin 1 (Sirt1) [27] also did not increase by exercise (Figure 4).

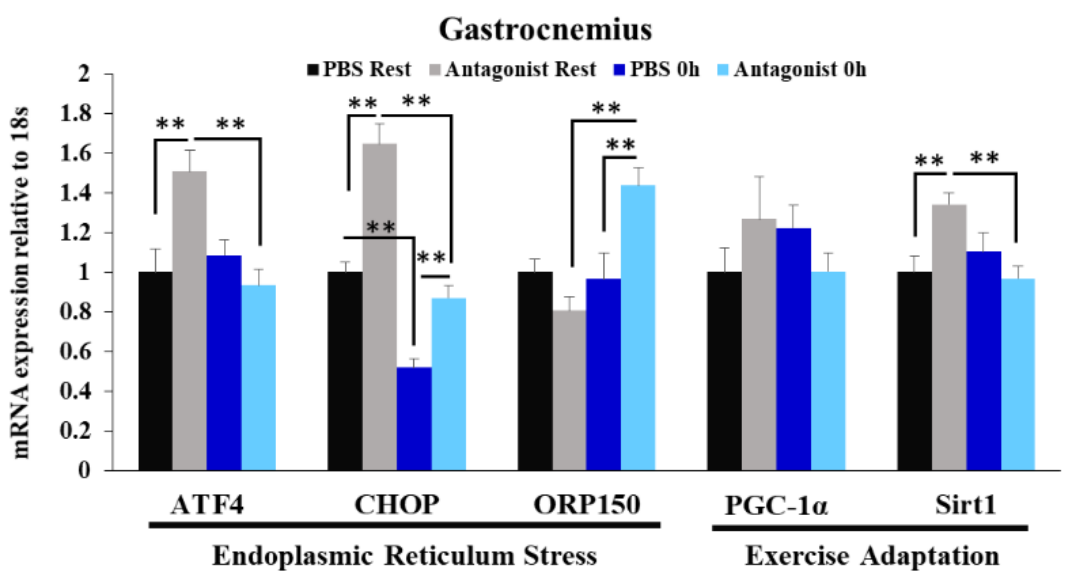

Figure 4. Gene expression of endoplasmic reticulum stress marker and inducing exercise adaptation $(n=6)$. Data are shown as mean \pm SE. Data were analyzed using two-way ANOVA followed by Bonferroni post hoc test $(* * p<0.01)$. ATF4, activating transcription factor 4 ; CHOP, C/EBP homologous protein; ORP150, oxygen-regulated protein 150; PGC-1 $\alpha$, peroxisome proliferatoractivated receptor coactivator- $1 \alpha$; Sirt1, sirtuin 1; ANOVA, analysis of variance; SE, standard error.

\subsection{Inhibition of CCR2 Signaling Exacerbates Exercise-Induced Inflammation in Kidney, Liver, and Adipose Tissues}

It is known that strenuous exercise induces internal organ damage and inflammation [7]. Hence, we next investigated the effects of CCR2 signaling inhibition on exerciseinduced inflammation in kidney, liver, and adipose tissues. In the kidney, exercise with CCR2 antagonist administration induced MCP-1 gene expression but did not change MCP-1 protein concentrations (Figure 5A,B). Kidney IL-1 $\beta$ concentrations also showed no changes after exercise (Figure 5C). In the liver, exercise with CCR2 antagonist administration induced the gene expression of IL-6, IL-1 $\beta$, MCP-1, and MCP-2, but it did not change MCP-1 protein concentrations (Figure $5 \mathrm{D}, \mathrm{E}$ ). The concentrations of IL- $1 \beta$ in the liver showed an increasing trend after exercise with CCR2 antagonist administration (Figure 5F). In the adipose tissue, exercise with CCR2 antagonist administration induced MCP-3 gene expression but did not change the concentrations of MCP-1 and IL-1 $\beta$ proteins (Figure 5G-I). These results indicated that the inhibition of CCR2 signaling exacerbated exercise-induced inflammation in the kidney, liver, and adipose tissues. 

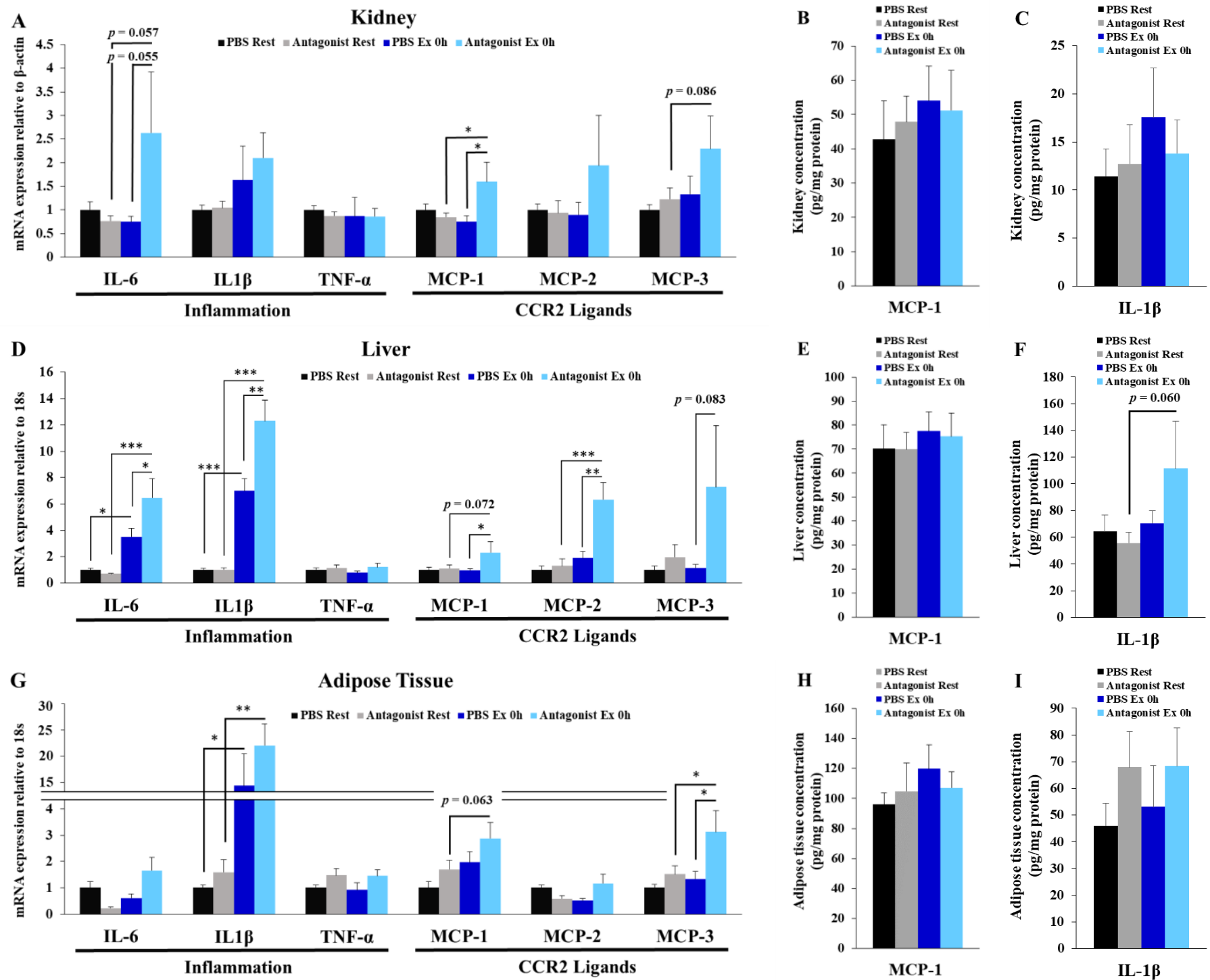

Figure 5. Gene expression of inflammation in (A) kidney, (D) liver and (G) adipose tissues $(n=6$, in each tissue). MCP-1 concentrations in (B) kidney, $(\mathbf{E})$ liver and $(\mathbf{H})$ adipose tissues $(n=6$, in each tissue). IL-1 $\beta$ concentrations in (C) kidney, (F) liver and (I) adipose tissues $(n=6$, in each tissue). Data are shown as mean \pm SE. Data were analyzed using two-way ANOVA followed by Bonferroni post hoc test $\left({ }^{*} p<0.05,{ }^{* *} p<0.01,{ }^{* * *} p<0.001\right)$. IL, interleukin; TNF- $\alpha$, tumor necrosis factor- $\alpha$; $\mathrm{MCP}$, monocyte chemoattractant protein; ANOVA, analysis of variance; SE, standard error.

\subsection{CCR2 Ligand-Producing Organs}

Although the inhibition of CCR2 signaling exacerbates exercise-induced inflammation, the mechanisms underlying this phenomenon are unidentified. Therefore, we hypothesized that increasing concentrations of CCR2 ligands in circulation regulate exercise-induced inflammation. We then focused on plasma MCP-1 concentrations before and immediately after exercise in the PBS-administrated group. However, we observed no changes in plasma MCP-1 concentrations after exercise in the PBS-administrated group (Figure 2C). We next hypothesized that tissue CCR2 ligands act in an autocrine manner. However, we found that the gene expressions of MCP-1, MCP-2, and MCP-3 in the gastrocnemius, kidney, liver, and adipose tissues did not change after exercise in the PBS-administrated group (Figures 2A and 5A,D,G). Moreover, MCP-1 protein concentrations in the kidney, liver, and adipose tissues showed no changes after exercise in the PBS-administrated group (Figure $5 \mathrm{~B}, \mathrm{E}, \mathrm{H}$ ). These results indicated that increasing concentrations of MCP-1 
in circulation and gastrocnemius, kidney, liver, and adipose tissues do not contribute to exercise-induced inflammation by the inhibition of CCR2 signaling. Increasing tissue concentrations of MCP-2 and MCP-3 proteins may also not contribute to exercise-induced inflammation by the inhibition of CCR2 signaling.

\section{Discussion}

In the present study, in contrast to our expectation that the inhibition of CCR2 signaling inhibits exercise-induced macrophage infiltration, we observed that CCR2 signaling inhibition exacerbated macrophage infiltration following exercise. This result is probably because receptors other than CCR2 mediate macrophage infiltration. Macrophages express various chemokine receptors (e.g., CCR2, CCR5, CCR7, and CXCR1) $[17,18,23,28]$, and several studies have reported that CCR5 and CCR7 contribute to macrophage infiltration in the adipose tissue of obese mice [23,28]. However, in the acute muscle injury model, whether CCR5 and CCR7 contribute to macrophage infiltration remains unexplored. In the present study, the gene expressions of CCR5 and CCR5 ligands, MCP-3 and MIP- $1 \beta$, were increased $24 \mathrm{~h}$ after exercise with CCR2 antagonist administration. Therefore, CCR5 may mediate macrophage infiltration in this model. We did not measure CCR7 gene expression. However, CCR7 and CCR7 ligands may also mediate macrophage infiltration in this model.

We also found that CCR2 signaling inhibition exacerbated exercise-induced inflammation independently of neutrophil activation and oxidative stress immediately after exercise. However, a further concern is about those factors that regulate exercise-induced inflammation by the inhibition of CCR2 signaling. One hypothesis is that increasing levels of CCR2 ligands regulate exercise-induced inflammation. However, we could not identify increasing concentrations of CCR2 ligands by exercise. One possibility is that circulating MCP-2 or MCP-3 concentrations are important, which were not measured in the present study. Another possibility is that organs not investigated in the present study produce CCR2 ligands locally and regulate exercise-induced inflammation in the entire body. Therefore, further studies are required to identify the CCR2 ligands whose levels fluctuate following exercise in circulation and organs. Another hypothesis is that basal CCR2 signaling is essential to regulate exercise-induced inflammation. Because the activation of CCR2 signaling phosphorylates more than 200 proteins [29], inhibiting basal CCR2 signaling may also influence gene expression and protein phosphorylation. These genes or phosphorylated proteins may be essential for regulating exercise-induced inflammation. In the present study, CCR2 antagonist administration alone also regulated the gene expression of GST, ATF4, CHOP, and Sirt1. Further research is necessary to identify key factors that trigger exercise-induced inflammation by CCR2 signaling.

Eotaxin is a natural antagonist for CCR2 [30,31]. Therefore, eotaxin may contribute to exercise-induced inflammation. However, several studies have reported that circulating eotaxin concentrations showed no changes following endurance exercise [32,33], and our unpublished data revealed that eotaxin/CCL11 gene expression does not change following exhaustive exercise in the mice gastrocnemius (data not shown). In contrast, cultured myotubes with electric pulse stimulation secrete eotaxin/CCL11 [15]. Therefore, local eotaxin in each organ may contribute to exercise-induced inflammation.

There is evidence showing that CCR2 antagonist administration improves atherosclerosis and type 2 diabetes $[34,35]$ and is expected to be an effective therapeutic agent. Nevertheless, CCR2 antagonist has not yet led to the development of medicines for the disease. Our findings may contribute to understanding the side effects of CCR2 antagonist.

\section{Limitation}

In the present study, we orally administrated the CCR2 antagonist RS-504393 to inhibit CCR2 signaling. However, we did not explore whether our administration protocol is appropriate to inhibit CCR2 signaling. Furuichi et al. have reported that the oral administration of the CCR2 antagonist RS-504393 protects ischemia-reperfusion injury in the kidney, and the same results were observed in CCR2-knockout mice [36]. Moreover, 
we observed the effects of CCR2 antagonist administration in the present study (e.g., exacerbating exercise-induced inflammation). On the basis of these results, we conclude that the inhibition of CCR2 signaling is successful. However, it is important to explore whether this protocol is appropriate for CCR2 signaling (e.g., the experiment whether RS-504393 administration inhibits the response of recombinant MCP-1 protein injection at several time points after RS-504393 administration).

In addition, RS-504393 exerts an antagonizing activity against the $\alpha 1$-adrenergic receptor $[37,38]$. Exercise increases the concentrations of circulating adrenalin, which modulates immune systems [1]. Therefore, the present study may also indicate the role of the $\alpha 1$-adrenergic receptor. Further studies are necessary using CCR2-knockout mice, or neutralizing antibodies for CCR2 ligands such as MCP-1, MCP-2, and MCP-3 are required to investigate the role of CCR2 signaling in exercise specifically.

\section{Conclusions}

Pharmacological inhibition of CCR2 signaling using RS-504393 exacerbated exerciseinduced inflammation independently of neutrophil infiltration and oxidative stress.

Supplementary Materials: The following supporting information can be downloaded at: https: //www.mdpi.com/article/10.3390/immuno2010003/s1, Figure S1: Gene expression of the housekeeping gene.

Author Contributions: Conceptualization, T.T.; investigation, T.T. and J.H.; supervision, K.S.; writingoriginal draft, T.T.; writing - review and editing, K.S. All authors have read and agreed to the published version of the manuscript.

Funding: This study was supported by a Grant-in-Aid for JSPS Research Fellow (20J21339) to T.T. from the Ministry of Education, Culture, Sports Science and Technology of Japan.

Institutional Review Board Statement: This study was conducted according to Guiding Principles for the Care and Use of Animals in the Academic Research Ethical Review Committee of Waseda University (2020-A29).

Informed Consent Statement: Not applicable.

Data Availability Statement: The raw data presented in this study are available upon request to first author.

Acknowledgments: The authors thank the laboratory members (Sihui Ma, Wonjun Choi and Mayu Araya) for technical support.

Conflicts of Interest: The authors declare no conflict of interest.

\section{References}

1. Gleeson, M.; Bishop, N.C.; Stensel, D.J.; Lindley, M.R.; Mastana, S.S.; Nimmo, M.A. The Anti-Inflammatory Effects of Exercise: Mechanisms and Implications for the Prevention and Treatment of Disease. Nat. Rev. Immunol. 2011, 11, 607-615. [CrossRef] [PubMed]

2. Safdar, A.; Saleem, A.; Tarnopolsky, M.A. The Potential of Endurance Exercise-Derived Exosomes to Treat Metabolic Diseases. Nat. Rev. Endocrinol. 2016, 12, 504-517. [CrossRef] [PubMed]

3. Sylow, L.; Kleinert, M.; Richter, E.A.; Jensen, T.E. Exercise-Stimulated Glucose Uptake-Regulation and Implications for Glycaemic Control. Nat. Rev. Endocrinol. 2017, 13, 133-148. [CrossRef] [PubMed]

4. Chang, Y.K.; Labban, J.D.; Gapin, J.I.; Etnier, J.L. The Effects of Acute Exercise on Cognitive Performance: A Meta-Analysis. Brain Res. 2012, 1453, 87-101. [CrossRef] [PubMed]

5. Suzuki, K.; Nakaji, S.; Yamada, M.; Totsuka, M.; Sato, K.; Sugawara, K. Systemic Inflammatory Response to Exhaustive Exercise. Cytokine Kinetics. Exerc. Immunol. Rev. 2002, 8, 6-48. [PubMed]

6. Suzuki, K.; Nakaji, S.; Yamada, M.; Liu, Q.; Kurakake, S.; Okamura, N.; Kumae, T.; Umeda, T.; Sugawara, K. Impact of a Competitive Marathon Race on Systemic Cytokine and Neutrophil Responses. Med. Sci. Sports Exerc. 2003, 35, 348-355. [CrossRef] [PubMed]

7. Suzuki, K.; Tominaga, T.; Ruhee, R.T.; Ma, S. Characterization and Modulation of Systemic Inflammatory Response to Exhaustive Exercise in Relation to Oxidative Stress. Antioxidants 2020, 9, 401. [CrossRef] 
8. Tominaga, T.; Ma, S.; Sugama, K.; Kanda, K.; Omae, C.; Choi, W.; Hashimoto, S.; Aoyama, K.; Yoshikai, Y.; Suzuki, K. Changes in Urinary Biomarkers of Organ Damage, Inflammation, Oxidative Stress, and Bone Turnover Following a 3000-m Time Trial. Antioxidants 2021, 10, 79. [CrossRef]

9. Tominaga, T.; Ikemura, T.; Yada, K.; Kanda, K.; Sugama, K.; Ma, S.; Choi, W.; Araya, M.; Huang, J.; Nakamura, N.; et al. The Effects of Beverage Intake after Exhaustive Exercise on Organ Damage, Inflammation and Oxidative Stress in Healthy Males. Antioxidants 2021, 10, 866. [CrossRef]

10. Tominaga, T.; Ma, S.; Saitou, K.; Suzuki, K. Glucose Ingestion Inhibits Endurance Exercise-Induced IL-6 Producing Macrophage Infiltration in Mice Muscle. Nutrients 2019, 11, 1496. [CrossRef]

11. Kawanishi, N.; Mizokami, T.; Niihara, H.; Yada, K.; Suzuki, K. Neutrophil Depletion Attenuates Muscle Injury after Exhaustive Exercise. Med. Sci. Sports Exerc. 2016, 48, 1917-1924. [CrossRef]

12. Kawanishi, N.; Mizokami, T.; Niihara, H.; Yada, K.; Suzuki, K. Macrophage Depletion by Clodronate Liposome Attenuates Muscle Injury and Inflammation Following Exhaustive Exercise. Biochem. Biophys. Rep. 2016, 5, 146-151. [CrossRef]

13. Miyatake, S.; Bilan, P.J.; Pillon, N.J.; Klip, A. Contracting C2C12 Myotubes Release CCL2 in an NF-KB-Dependent Manner to Induce Monocyte Chemoattraction. Am. J. Physiol. Endocrinol. Metab. 2016, 310, E160-E170. [CrossRef]

14. Evers-van Gogh, I.J.A.; Alex, S.; Stienstra, R.; Brenkman, A.B.; Kersten, S.; Kalkhoven, E. Electric Pulse Stimulation of Myotubes as an In Vitro Exercise Model: Cell-Mediated and Non-Cell-Mediated Effects. Sci. Rep. 2015, 5, 10944. [CrossRef]

15. Chen, W.; Nyasha, M.R.; Koide, M.; Tsuchiya, M.; Suzuki, N.; Hagiwara, Y.; Aoki, M.; Kanzaki, M. In Vitro Exercise Model Using Contractile Human and Mouse Hybrid Myotubes. Sci. Rep. 2019, 9, 11914. [CrossRef]

16. Martinez, C.O.; McHale, M.J.; Wells, J.T.; Ochoa, O.; Michalek, J.E.; McManus, L.M.; Shireman, P.K. Regulation of Skeletal Muscle Regeneration by CCR2-Activating Chemokines Is Directly Related to Macrophage Recruitment. Am. J. Physiol. Regul. Integr. Comp. Physiol. 2010, 299, R832-R842. [CrossRef]

17. Saini, J.; McPhee, J.S.; Al-Dabbagh, S.; Stewart, C.E.; Al-Shanti, N. Regenerative Function of Immune System: Modulation of Muscle Stem Cells. Ageing Res. Rev. 2016, 27, 67-76. [CrossRef]

18. Warren, G.L.; Hulderman, T.; Mishra, D.; Gao, X.; Millecchia, L.; O’Farrell, L.; Kuziel, W.A.; Simeonova, P.P. Chemokine Receptor CCR2 Involvement in Skeletal Muscle Regeneration. FASEB J. Off. Publ. Fed. Am. Soc. Exp. Biol. 2005, 19, 413-415. [CrossRef]

19. Blanc, R.S.; Kallenbach, J.G.; Bachman, J.F.; Mitchell, A.; Paris, N.D.; Chakkalakal, J.V. Inhibition of Inflammatory CCR2 Signaling Promotes Aged Muscle Regeneration and Strength Recovery after Injury. Nat. Commun. 2020, 11, 4167. [CrossRef]

20. Kamei, N.; Tobe, K.; Suzuki, R.; Ohsugi, M.; Watanabe, T.; Kubota, N.; Ohtsuka-Kowatari, N.; Kumagai, K.; Sakamoto, K.; Kobayashi, M.; et al. Overexpression of Monocyte Chemoattractant Protein-1 in Adipose Tissues Causes Macrophage Recruitment and Insulin Resistance. J. Biol. Chem. 2006, 281, 26602-26614. [CrossRef]

21. Sell, H.; Dietze-Schroeder, D.; Kaiser, U.; Eckel, J. Monocyte Chemotactic Protein-1 Is a Potential Player in the Negative Cross-Talk between Adipose Tissue and Skeletal Muscle. Endocrinology 2006, 147, 2458-2467. [CrossRef] [PubMed]

22. Kawashima, M.; Kawanishi, N.; Tominaga, T.; Suzuki, K.; Miyazaki, A.; Nagata, I.; Miyoshi, M.; Miyakawa, M.; Sakuraya, T.; Sonomura, T.; et al. Icing after Eccentric Contraction-Induced Muscle Damage Perturbs the Disappearance of Necrotic Muscle Fibers and Phenotypic Dynamics of Macrophages in Mice. J. Appl. Physiol. Bethesda Md 1985 2021, 130, 1410-1420. [CrossRef] [PubMed]

23. Kitade, H.; Sawamoto, K.; Nagashimada, M.; Inoue, H.; Yamamoto, Y.; Sai, Y.; Takamura, T.; Yamamoto, H.; Miyamoto, K.; Ginsberg, H.N.; et al. CCR5 Plays a Critical Role in Obesity-Induced Adipose Tissue Inflammation and Insulin Resistance by Regulating Both Macrophage Recruitment and M1/M2 Status. Diabetes 2012, 61, 1680-1690. [CrossRef] [PubMed]

24. Ahmed, S.M.U.; Luo, L.; Namani, A.; Wang, X.J.; Tang, X. Nrf2 Signaling Pathway: Pivotal Roles in Inflammation. Biochim. Biophys. Acta Mol. Basis Dis. 2017, 1863, 585-597. [CrossRef]

25. Kobayashi, E.H.; Suzuki, T.; Funayama, R.; Nagashima, T.; Hayashi, M.; Sekine, H.; Tanaka, N.; Moriguchi, T.; Motohashi, H.; Nakayama, K.; et al. Nrf2 Suppresses Macrophage Inflammatory Response by Blocking Proinflammatory Cytokine Transcription. Nat. Commun. 2016, 7, 11624. [CrossRef]

26. Hasnain, S.Z.; Lourie, R.; Das, I.; Chen, A.C.-H.; McGuckin, M.A. The Interplay between Endoplasmic Reticulum Stress and Inflammation. Immunol. Cell Biol. 2012, 90, 260-270. [CrossRef]

27. Egan, B.; Zierath, J.R. Exercise Metabolism and the Molecular Regulation of Skeletal Muscle Adaptation. Cell Metab. 2013, 17, 162-184. [CrossRef]

28. Hellmann, J.; Sansbury, B.E.; Holden, C.R.; Tang, Y.; Wong, B.; Wysoczynski, M.; Rodriguez, J.; Bhatnagar, A.; Hill, B.G.; Spite, M. CCR7 Maintains Nonresolving Lymph Node and Adipose Inflammation in Obesity. Diabetes 2016, 65, 2268-2281. [CrossRef]

29. Huang, C.; Foster, S.R.; Shah, A.D.; Kleifeld, O.; Canals, M.; Schittenhelm, R.B.; Stone, M.J. Phosphoproteomic Characterization of the Signaling Network Resulting from Activation of the Chemokine Receptor CCR2. J. Biol. Chem. 2020, 295, 6518-6531. [CrossRef]

30. Ogilvie, P.; Bardi, G.; Clark-Lewis, I.; Baggiolini, M.; Uguccioni, M. Eotaxin Is a Natural Antagonist for CCR2 and an Agonist for CCR5. Blood 2001, 97, 1920-1924. [CrossRef]

31. Ogilvie, P.; Paoletti, S.; Clark-Lewis, I.; Uguccioni, M. Eotaxin-3 Is a Natural Antagonist for CCR2 and Exerts a Repulsive Effect on Human Monocytes. Blood 2003, 102, 789-794. [CrossRef] 
32. Schild, M.; Eichner, G.; Beiter, T.; Zügel, M.; Krumholz-Wagner, I.; Hudemann, J.; Pilat, C.; Krüger, K.; Niess, A.M.; Steinacker, J.M.; et al. Effects of Acute Endurance Exercise on Plasma Protein Profiles of Endurance-Trained and Untrained Individuals over Time. Mediat. Inflamm. 2016, 2016, 4851935. [CrossRef]

33. Little, H.C.; Tan, S.Y.; Cali, F.M.; Rodriguez, S.; Lei, X.; Wolfe, A.; Hug, C.; Wong, G.W. Multiplex Quantification Identifies Novel Exercise-Regulated Myokines/Cytokines in Plasma and in Glycolytic and Oxidative Skeletal Muscle *. Mol. Cell. Proteom. 2018, 17, 1546-1563. [CrossRef]

34. Gilbert, J.; Lekstrom-Himes, J.; Donaldson, D.; Lee, Y.; Hu, M.; Xu, J.; Wyant, T.; Davidson, M. Effect of CC Chemokine Receptor 2 CCR2 Blockade on Serum C-Reactive Protein in Individuals at Atherosclerotic Risk and With a Single Nucleotide Polymorphism of the Monocyte Chemoattractant Protein-1 Promoter Region. Am. J. Cardiol. 2011, 107, 906-911. [CrossRef]

35. White, G.E.; Iqbal, A.J.; Greaves, D.R. CC Chemokine Receptors and Chronic Inflammation-Therapeutic Opportunities and Pharmacological Challenges. Pharmacol. Rev. 2013, 65, 47-89. [CrossRef]

36. Furuichi, K.; Wada, T.; Iwata, Y.; Kitagawa, K.; Kobayashi, K.-I.; Hashimoto, H.; Ishiwata, Y.; Asano, M.; Wang, H.; Matsushima, K.; et al. CCR2 Signaling Contributes to Ischemia-Reperfusion Injury in Kidney. J. Am. Soc. Nephrol. 2003, 14, 2503-2515. [CrossRef]

37. Mirzadegan, T.; Diehl, F.; Ebi, B.; Bhakta, S.; Polsky, I.; McCarley, D.; Mulkins, M.; Weatherhead, G.S.; Lapierre, J.M.; Dankwardt, J.; et al. Identification of the Binding Site for a Novel Class of CCR2b Chemokine Receptor Antagonists: Binding to a Common Chemokine Receptor Motif within the Helical Bundle. J. Biol. Chem. 2000, 275, 25562-25571. [CrossRef]

38. DeSantis, A.J.; Enten, G.A.; Gao, X.; Majetschak, M. Chemokine Receptor Antagonists with A1-Adrenergic Receptor Blocker Activity. J. Basic Clin. Physiol. Pharmacol. 2021. [CrossRef] 